\title{
La gestión en primarias multigrado indígenas inscritas al PETc: caso comparativo
}

\section{Management in Multigrade Indigenous Elementary Schools Enrolled in the PETC: A Comparative Case Study}

\author{
Juan Páez Cárdenas (*) https://orcid.org/0000-0001-8991-0112 \\ María Guadalupe Tinajero Villavicencio (*) https://orcid.org/0000-0002-3120-9099
}

(*) Universidad Autónoma de Baja California

(Recibido: 10 de noviembre de 2019; Aceptado para su publicación: 11 de marzo de 2020)

Cómo citar: Páez, J. y Tinajero, M. G. (2020). La gestión en primarias multigrado indígenas inscritas al PETc: caso comparativo. Revista Electrónica de Investigación Educativa, 22, e29, 1-14. https://doi.org/10.24320/redie.2020.22.e29.3564

\section{Resumen}

El objetivo del estudio fue explorar los distintos aspectos que inciden en la gestión de dos primarias multigrado del servicio indígena, ambas participantes en el Programa de Escuelas de Tiempo Completo (PETC) en Baja California, México. Se empleó el método de estudio de caso de tipo comparativo desde la perspectiva etnográfica para observar dos escuelas ubicadas en dos ciudades. Los hallazgos revelan que el número, la condición laboral y permanencia de los docentes son elementos diferenciadores que inciden en la gestión de cada plantel. Además, si bien la inyección de recursos económicos y el servicio de alimentación proporcionados por el programa beneficia a las comunidades escolares, la mejora en el servicio educativo no se logra del todo debido a que el PETC incumple su propia normativa y no contempla la totalidad de dimensiones que atraviesan la gestión.

Palabras clave: Población indígena, Enseñanza primaria, Condiciones de empleo del docente, Gestión educacional.

\begin{abstract}
The objective of this study was to explore the various aspects that affect the management of two multigrade elementary schools in Baja California, Mexico, both operating under the "indigenous service" system and part of the Full-Time Schools Program (PETC). A comparative case study was conducted from an ethnographic perspective to observe two schools in two cities. The findings show that the number of teachers, their employment status and their long-term presence at the school are factors that impact management. Furthermore, while school communities have benefited from the cash injections and food service provided by the program, improvements in the education service cannot be fully realized due to a failure by the PETC to comply with its own regulations and the fact that the program does not take into account all the dimensions of management.
\end{abstract}

Keywords: Indigenous populations, Elementary education, Teacher conditions of employment; Educational management.

\section{Introducción}

Existen muchas definiciones y usos del término "gestión" en el ámbito educativo, además de una cantidad considerable de literatura especializada que lo utiliza para referir un sinfín de tareas propias de la escuela. 
Un antecedente para entender cabalmente su proliferación se vincula con la lógica que irrumpió en la administración pública durante la década de los ochenta (Smyth, 2001), conocida como New public management. Su práctica y promoción inició en países anglosajones y Tolofari (2005, p. 84) precisa que el concepto que cobró fuerza fue precisamente el de management, que combinado con la palabra school se tradujo al español como "gestión escolar".

La propuesta impulsada desde el school management se centró en la mejora de los centros educativos (Caldwell, 2005; Gertler et al., 2007 y unESCO-IIEP, 2019); en ese sentido, debe entenderse su repercusión en las iniciativas de política educativa durante la década de los noventa en México, como lo documenta Bonilla (2008), quien puntualiza cómo la noción de gestión escolar, además, se vinculó con la de calidad educativa y que estos dos conceptos se volvieron referentes para procurar la mejora educativa (Bonilla, 2008, p. 2). Otros desarrollos teóricos, como el de Ezpeleta (1992a), insisten en que la noción de gestión en el campo educativo se relacionaba mayormente con la dimensión administrativa de la escuela, sin considerar otras dimensiones implicadas en ella. A partir de este razonamiento, la autora presenta una noción de gestión que da cuenta de la multiplicidad de componentes que inciden en la generación de condiciones para el fin fundamental de la escuela, es decir, para promover los procesos de enseñanza y aprendizaje:

Si la gestión puede entenderse como el conjunto de estrategias para acercar las formas de operación institucional del servicio educativo a la finalidad específica de la escuela -ofrecer enseñanza y provocar aprendizajes-, se estaría revitalizando la centralidad de la enseñanza en su movimiento institucional, del mismo modo que se estaría introduciendo la aceptación del dinamismo y la diversidad que caracterizan a los establecimientos escolares (Ezpeleta, 1997, p. 102).

Así, para observar la gestión no basta con dar cuenta de los procesos administrativos o de la normativa dictada desde las instancias del sistema educativo, es necesario, más bien, ampliar la mirada para detectar la diversidad de procesos sociales que inciden en la existencia diaria de las escuelas, desde la participación de los padres hasta las condiciones materiales y de financiamiento escolar; todos ellos como elementos involucrados en la generación de las condiciones para la enseñanza. Estos aspectos pueden ser diferentes para cada plantel, dependiendo de sus características contextuales y organizativas. Ezpeleta (1992a) plantea que, en el plano metodológico, la perspectiva etnográfica es la que presenta mayores posibilidades de aproximación a estos procesos inmersos en las realidades educativas.

La concepción de "gestión" de la autora se inserta en una amplia literatura en el ámbito educativo sobre el término. Una parte de esta producción académica lo relaciona directamente con la figura del director escolar y la noción de liderazgo (Githiari, 2017; Naidoo, 2019; Nava, 2018) o como el ordenamiento para conseguir la eficacia de las escuelas (Alfirevic et al., 2016; Butsankom et al., 2016; Mortimore et al., 1988).

Desde nuestra postura, la conceptualización amplia de Ezpeleta ofrece significativas posibilidades para comprender lo que subyace en la vida cotidiana de las escuelas y cómo la comunidad escolar resuelve las demandas, no sólo de las distintas instancias del sistema educativo, sino de los padres de familia, de los estudiantes y de los mismos docentes, sobre todo en escuelas multigrado que, por sus características, llegan a ser espacios complejos por las demandas de toda índole que imponen a los maestros.

En México, la escuela multigrado tiene una larga trayectoria. Sus inicios se remontan a la escuela rural de la década de los veinte. Desde entonces su organización se expandió, aunque su contenido se homogeneizó con el de las escuelas graduadas. Las estadísticas muestran que en el ciclo 2016-2017 cuatro de cada diez escuelas primarias continuaban siendo multigrado. Si revisamos las cifras por tipo de servicio educativo, entonces, el porcentaje cambia drásticamente, el $42.7 \%$ de las escuelas generales son multigrado, pero en el servicio indígena el porcentaje se incrementa a 65 (Instituto Nacional para la Evaluación de la Educación [INEE], 2019). Lo más revelador para entender la poca importancia que se le ha concedido a este tipo de organización es la ausencia de políticas específicas para el trabajo docente y la gestión.

El término multigrado alude a grupos de clases combinadas o clases de edades mixtas organizadas que tratan de dar respuesta a las necesidades y características de una población (Little, 2004). En la escuela 
multigrado un docente enseña dos o más grados de forma simultánea y, sin lugar a dudas, este tipo de atención forma parte de la realidad educativa en muchos países tanto en vías de desarrollo como desarrollados (Mejía et al., 2016; UNESCO, 2015). Para Blease y Condy (2015), la ubicación geográfica y la pobreza de la población parecen ser las variables que determinan las escuelas multigrado y, en América Latina, de acuerdo con Galván y Espinoza (2017) este tipo de organización escolar se extendió a poblaciones rurales y habitadas por pueblos originarios, sobre todo debido al reducido número de habitantes. En cambio, en otros contextos se considera "una oportunidad para la innovación didáctica y el trabajo colaborativo entre docentes" (Arriagada y Calzadilla, 2017, p. 92).

No abundan trabajos que indaguen, particularmente, la gestión que desarrollan este tipo de planteles. Consideramos que nuestro estudio puede aportar de manera significativa -si bien, no generalizable- al conocimiento de esta cuestión. El objetivo fue explorar y comparar los distintos elementos en el día a día de las escuelas que inciden en la gestión de dos primarias multigrado de servicio indígena en Baja California inscritas al PETC e indagar su relación con el logro educativo de cada una, aspecto que repercute en la valoración de las escuelas.

\subsection{Contexto de la investigación}

Una de las características más sobresalientes de las escuelas indígenas en Baja California es la población que atiende; de 67 escuelas, cinco se ubican en comunidades de los grupos originarios del estado; el resto recibe, en una gran mayoría, a los hijos de jornaleros migrantes agrícolas indígenas provenientes de diferentes estados de la República, como Oaxaca, Chiapas, Guerrero y Michoacán. La mano de obra para trabajo en el campo y la migración constante de familias requirió, por parte del sistema educativo estatal, la atención a la demanda educativa de niños pertenecientes a diversos grupos. En la actualidad, hay presencia de niños hablantes de 30 lenguas (SEP-Subsecretaría de Educación Básica-DGEI, 2019). Las escuelas están distribuidas en zonas rurales, periurbanas y urbanas; en el ciclo 2017-2018 se contabilizaron 10,807 niños inscritos y las actividades se desarrollaron con el apoyo de 434 docentes (INEE, 2019). Las autoridades estatales reportaron una eficiencia terminal de $97.10 \%, .42 \%$ de reprobación y .05\% de abandono escolar (SEP-Subsecretaría de Educación Básica-DGEl, 2019).

Del total de ellas, 35 participan en el Programa de Escuelas de Tiempo Completo (PETC), y 29.9\% son de organización multigrado (INEE, 2019). Un común denominador de todas las escuelas multigrado estatales es el bajo logro educativo, según los reportes de la estrategia evaluativa del Instituto Nacional para la Evaluación de la Educación (INEE) denominada Plan Nacional para la Evaluación de los Aprendizajes (PLANEA): en las escuelas primarias indígenas multigrado de Baja California, más del $50 \%$ de los alumnos obtiene un nivel de dominio insuficiente (SEP, s.f.).

Las escuelas que se observaron pertenecen al sistema federalizado de educación y se ubican en comunidades de media (Ensenada) y alta marginación (Tijuana). Se trata de planteles que fueron gestionados originalmente por las comunidades al inicio de su asentamiento en el área. La primaria de Ensenada atiende principalmente a hijos de jornaleros agrícolas, la mayor parte establecidos en la zona, mientras que la de Tijuana presta servicio a niños cuyos padres trabajan en la industria maquiladora o en oficios diversos. En el 2014 las escuelas se adscribieron al PETC, mediante el cual accedieron a recursos directos para mejoras de infraestructura y recursos educativos.

Debido a que el programa emite reglas de operación año con año, esta disposición ha permitido modificar los objetivos, la población beneficiada, el monto de los recursos económicos y los apoyos brindados a las escuelas. En 2018, por ejemplo, quedó establecido que el apoyo para las escuelas estaría vinculado a cuatro aspectos: fortalecimiento del modelo de tiempo completo (compensaciones al personal de tiempo completo, directores y docentes, y mejora de los espacios escolares); fortalecimiento de la autonomía de gestión escolar (entrega de 90 mil pesos -sujeto a disponibilidad presupuestaria-y de materiales, asesorías y acondicionamiento de espacios); apoyos para el servicio de alimentación; y apoyos para la implementación local (seguimiento y evaluación del programa). El PETC anotó que el objetivo era beneficiar a poblaciones en condiciones desfavorables que asistían a escuelas públicas de educación básica en contextos urbanomarginales, indígenas o migrantes (Acuerdo 21/12/17). 


\section{Método}

Este es un estudio de caso de tipo comparativo como lo propone Parcerisa (2016), elaborado desde la perspectiva etnográfica. El interés fue explorar y comparar, desde la cotidianidad de las escuelas, las características de la gestión de dos primarias multigrado. El trabajo aborda, principalmente, aspectos de la gestión relacionados con las condiciones materiales de las escuelas, sus horarios de servicio, el financiamiento escolar vinculado al PETC y las condiciones laborales de los equipos docentes.

La selección de las dos escuelas, ubicadas en zonas periurbanas de Ensenada y Tijuana, responde a que -a pesar de presentar varios aspectos similares (ser primarias multigrado, de servicio indígena, periurbanas, con una matrícula menor a 76 estudiantes, inscritas al PETC)-, ambas presentaban, según los datos arrojados por la prueba PLANEA, trayectorias contrarias.

En las evaluaciones, que iniciaron en el 2015 y se repitieron en el 2018, la primaria de Tijuana había aumentado el número de alumnos en el nivel I de logro educativo, es decir, el más bajo, tanto en el campo de Lenguaje y Comunicación como en el de Matemáticas. Por el contrario, la escuela de Ensenada había tenido una significativa disminución de estudiantes en ese nivel en ambos campos (SEP, 2020).

Se realizaron dos estancias de observación en cada escuela. Una en la primera mitad del ciclo escolar (octubre-noviembre de 2018) y la segunda al final (mayo-junio de 2019). La primera fue de nueve días y la segunda de cinco. También se observó una sesión de Consejo Técnico de cada escuela. Durante ese período permanecimos en la escuela durante toda la jornada escolar (8:00 a 15:00 o 16:00 horas). El plan de observación fue amplio: incluyó horarios de entrada y salida de las escuelas, características generales de la población estudiantil, financiamiento escolar, condiciones operativas del PETc, requerimientos de capacitación a docentes, rendición de cuentas de los directores, condiciones laborales del equipo docente, infraestructura escolar, enseñanza de la lengua indígena, convivencia escolar, grado de asistencia estudiantil, participación de los padres de familia en la gestión, la presencia de docentes contratados bajo el esquema del PETC y el contexto comunitario. En los consejos técnicos observamos la interacción de los equipos docentes de las escuelas con otras primarias de la zona escolar y registramos la discusión en torno a la elaboración de la ruta de mejora.

Entrevistamos a los distintos actores de ambos espacios escolares (maestros, directores, supervisores, padres de familia, estudiantes, maestros del PETC, figuras clave de las comunidades) con entrevistas no estructuradas y semiestructuradas, además de mantener pláticas informales con ellos. Los lugares de las entrevistas fueron, en su mayoría, dentro de las escuelas o la supervisión de una de ellas. Sin embargo, también fueron importantes las conversaciones libres mantenidas al acompañar a los maestros al salir de la escuela.

Desde la perspectiva etnográfica, y como sostiene Rockwell (2009, p. 48), consideramos que "la constante observación e interacción en una localidad" proporciona mayor posibilidad de comprensión de lo que subyace en los distintos procesos socioculturales.

Por otra parte, aplicamos el cuestionario de Evaluación de Condiciones Básicas para la Enseñanza y el Aprendizaje (ECEA) (INEE, s.f.) al inicio de las visitas. Este cuestionario consta de siete dimensiones: infraestructura, mobiliario y equipo, material de apoyo educativo, personal de la escuela, gestión del aprendizaje, organización escolar, y convivencia. Además, congruentes con la metodología propuesta recabamos documentos de gestión, como la Ruta de Mejora Escolar. Esta información fue de utilidad para contextualizar la observación y el análisis posterior.

Para analizar los datos se empleó el software para análisis de datos cualitativos Atlas.ti, con el cual realizamos la codificación no exhaustiva de los diarios de campo y las entrevistas, para posteriormente efectuar un análisis etnográfico (Rockwell, 2009) de todo el material.

Al enfocarnos en los temas de gestión no se abordaron directamente los aspectos pedagógicos dentro de las aulas, las particularidades de los aspectos multinivel ni las condiciones de interculturalidad, por lo que 
son esas algunas limitantes de este estudio.

\section{Resultados 3.1 La primaria en Tijuana}

Desde la década de los sesenta, Tijuana -ciudad que comparte la frontera con San Ysidro, Californiapresentó un acelerado crecimiento de la industria maquiladora, que se instaló en parques industriales por toda la ciudad, pero principalmente en la parte Noreste y Este. Con la llegada de esta industria se crearon grandes asentamientos de vivienda precaria habitados por olas de migrantes que se quedaron a trabajar en las maquilas.

La escuela se ubica en la zona este de la ciudad y asisten niños de un conglomerado de alrededor de 250 hogares (los habitantes trabajaban mayormente en las fábricas o maquiladoras de las cercanías, como vendedores ambulantes o como choferes del transporte público; otros trabajadores lo hacían del otro lado de la frontera). La mayor parte de las viviendas son precarias. El único acceso al asentamiento es un camino de terracería, bastante accidentado en la parte superior de un cerro. No existe ningún tipo de transporte público que llegue hasta el lugar. Los habitantes deben usar automóviles o subir y bajar a pie. El único servicio público disponible es la energía eléctrica. El agua deben comprarla a camiones-cisterna.

La primaria fue fundada por la gente de la comunidad en el 2006. Como muchas otras escuelas de México, la institución tuvo sus orígenes en cuartos prestados de las viviendas de las familias lugareñas y con un maestro enviado por el sistema educativo para dar servicio a los habitantes del incipiente caserío. Una congregación evangélica estadounidense apoyó a los pobladores a construir sus casas. La misma asociación fue la que levantó las primeras aulas de la escuela.

Durante los primeros cuatro años esta escuela no fue reconocida, sino que, para su operación, tanto maestros como alumnos estaban adscritos a otra primaria de Tijuana, funcionando como una escuelaextensión. A decir del asesor técnico pedagógico de la zona, quien fungió como director de la escuela en el ciclo 2010-2011, el plantel se caracterizó por presentar un equipo docente inestable, con muchos cambios. Del ciclo escolar 2014-2015 al 2017-2018, pasaron por la escuela 14 maestros. Algunos de ellos tenían una plaza base, mientras que otros eran interinos.

Los resultados de la prueba PLANEA para el ciclo 2017-2018 de esta escuela -en la cual participó una muestra de estudiantes de sexto grado y cuya aplicación estuvo a cargo de personal externo a la escuelaevidenciaron una insuficiencia en los aprendizajes para el campo de Lengua y Comunicación: el 44.4\% de alumnos se ubicó en el nivel más bajo y un $66.7 \%$ en el mismo nivel para Matemáticas; en el ciclo 20142015, los resultados de dicha prueba habían sido más prometedores: 30\% y 60\%, respectivamente.

La infraestructura de esta escuela cuenta con cuatro salones y un comedor de madera construidos por la congregación evangélica. Además, existen dos salones de concreto, uno de ellos considerado "aula digna", cuya construcción fue realizada por las autoridades educativas correspondientes, mientras que los sanitarios fueron edificados por instancias del gobierno del estado. Los dos salones de concreto eran los utilizados como salones de clase. El resto era muy poco -o nada- utilizado. En la entrada había una pequeña cancha de usos múltiples. El plantel no contaba con el servicio de Internet.

La matrícula oficial era de 53 alumnos. Tanto los maestros como los padres de familia entrevistados coincidieron en que no existían, al momento de la observación, niños reconocidos como hablantes de lengua indígena en la comunidad. Sin embargo, en el formato denominado Perfil lingüístico de escuela -el cual había sido elaborado a inicios del ciclo escolar por el director- se registró a nueve niños hablantes de lengua indígena: cinco del maya; dos del mixteco alto y dos de purépecha. Si bien el director aclaró que se trataba de niños de ascendencia indígena y no de hablantes, agregó que la mayoría de ellos había nacido en Tijuana. En la interacción con la población estudiantil no detectamos a ningún niño que hablara una lengua distinta al español.

La planta docente se componía de dos docentes (mujer y hombre), cada uno a cargo de un grupo 
multigrado. La maestra atendía a los niños de primero a tercero, mientras que el profesor se ocupaba del grupo de cuarto a sexto. Él, además, fungía como encargado de la dirección escolar del período regular (de 8 de la mañana a 12 de la tarde). Había también otra profesora como "directora de tiempo completo" (de 12 a 4 de la tarde). Todos contratados de forma interina. El interinato les impedía elaborar planes de gestión a largo plazo, puesto que era incierto el tiempo que podrían permanecer en el plantel.

El director se reconocía a sí mismo como indígena purépecha y se había formado en una Normal del estado de Michoacán. La maestra comentó haber estudiado una licenciatura en la principal universidad pública de Baja California, mientras que la directora de tiempo completo había egresado recientemente de la Licenciatura en Ciencias de la Educación de una universidad privada. Ninguna de las dos se reconocía como indígena.

Después de unos días de visitar la escuela, el equipo docente se redujo. A la maestra no le fue renovado su interinato tras las airadas quejas de varias madres de familia ante la dirección y supervisión escolar, quienes evaluaron de forma negativa su trabajo en el aula. Las madres no pedían el despido de la profesora, pero sí un cambio sustantivo en su desempeño. Ante esta situación, la directora de tiempo completo obtuvo un contrato de interinato y se quedó con el grupo de primero a tercero, además de continuar como directora. La concepción de Ezpeleta (1992b, p. 29) sobre la trama escolar que considera "las relaciones y procesos que cotidianamente constituyen y expresan su institucionalidad" nos ayuda a observar aquí una acción instituyente en la gestión de un actor distinto a los docentes o autoridades educativas.

El financiamiento que esta primaria obtuvo durante el ciclo escolar 2018-2019 (exceptuando el pago a los maestros-directores) fue de 22,300 pesos del Programa Integral de Mantenimiento de Escuelas (PIME), y por parte del PETC recibió dos cantidades: 40,000 pesos en octubre, y 50,000 a finales del ciclo escolar. Para la compra de comida recibió el equivalente a 13 pesos por cada estudiante inscrito multiplicado por los días hábiles del calendario escolar. Además del pago de 3,800 pesos mensuales asignado a la madre de familia que fungía como coordinadora del comedor.

Los recursos del PIME y del PETC fueron utilizados, principalmente, para mejorar la infraestructura escolar: nuevas lámparas en la escuela, una estufa e instalación de piso de loseta en el comedor, además de una bocina de alto volumen.

Los maestros que habían trabajado en la escuela en ciclos anteriores eran mixtecos y habían enseñado nociones de su lengua a los niños. Sin embargo, el director de la escuela decidió no enseñar esa lengua indígena debido a que la desconocía y pensó - dijo- que intentar enseñar el purépecha (del cual se describió como hablante activo), no era buena idea, ya que representaría una disrupción en la secuencia de aprendizaje de los alumnos. En este escenario, durante el ciclo escolar 2018-2019 no se impartió la enseñanza de la lengua indígena señalada en los planes y programas de este servicio. Por otra parte, se carecía de un modelo educativo oficial acorde a la escuela multigrado.

A pesar de que el PETC señala la hora de salida entre 3 y 4 de la tarde, en esta escuela la pregunta obligada de las madres de familia era "¿a qué hora saldrán los niños hoy?". Durante los días de observación, la hora de salida fue muy irregular debido, principalmente, a trámites administrativos y cursos de capacitación que los docentes debían realizar fuera de la escuela. Las jornadas con un horario completo no alcanzaron a representar un tercio del total. Esto se debió también a las faltas de la maestra a la que no le fue renovado el interinato. Los días que salían antes del horario reglamentario los docentes se aseguraban de que los niños recibieran el servicio de alimento del PETC antes de irse.

Al no contar con una computadora con Internet en la escuela, el director se trasladaba con frecuencia a la supervisión a llenar formatos o llevar y traer materiales. Los cursos de capacitación fuera de la escuela (como los relacionados al PETC o los que incluyeron a los padres de familia) implicaban suspender actividades al tratarse de dos maestros únicamente. 
En suma, en esta primaria los componentes de la gestión no pedagógicos repercutían en las condiciones para la enseñanza y el cumplimiento con los tiempos de trabajo. El servicio educativo estaba atravesado por la falta de conectividad, las cargas administrativas, los requerimientos de capacitación y las condiciones laborales de los dos únicos maestros, quienes debían ejercer funciones directivas al mismo tiempo que eran profesores frente a grupo. Además, el hecho de que -en el momento de la observación- en esta primaria no se adjudicaran plazas base, la condenaba a una rotación docente continua en detrimento del trabajo pedagógico.

\subsection{La primaria en Ensenada}

La primaria multigrado ubicada en la periferia de Ensenada fue fundada en el año de 1992. Está enclavada en un asentamiento de indígenas mixtecos, quienes llegaron a la zona -a partir de la década de los ochentaprovenientes del estado de Oaxaca con la intención de emplearse como jornaleros de los campos agrícolas de la localidad. A últimas fechas, la zona donde está la escuela ha tenido un auge económico significativo, ya que se ha detonado como zona turística a raíz del auge de empresas vinícolas y gastronómicas.

Gradualmente, las gestiones de la comunidad consiguieron que se introdujeran servicios públicos a la localidad. En el momento de la observación (noviembre 2018 y junio 2019), el lugar contaba con energía eléctrica, pavimento y agua potable. La escuela, además, tenía servicio de Internet. Los resultados de la prueba PLANEA del ciclo 2017-2018 para este plantel arrojaron 42.9\% de alumnos en el Nivel I para Lengua y Comunicación y 57.1 en el mismo nivel para Matemáticas. Los resultados habían mejorado de manera significativa con relación a las dos anteriores evaluaciones de esta prueba (2015 y 2016) (SEP, 2020).

En la primera estancia de observación la escuela registró 71 estudiantes. Había dos maestros varones frente a grupo y una profesora, quien, además de tener grupo, fungía como encargada de despacho en la dirección. Los profesores atendían el primero y tercer ciclo, mientras que la docente atendía el segundo. Después del mediodía, la maestra podía dedicarse a cuestiones administrativas, ya que en su lugar ingresaba un docente contratado por parte del PETC. A la escuela acudía también una persona que enseñaba nociones de inglés en los distintos grupos, de forma gratuita. Además, dos veces a la semana un maestro de Tae kwon do iba a la escuela a dar una clase a algunos niños; en este caso, los padres de familia eran quienes pagaban las clases.

Los tres maestros de grupo tenían una plaza base. Sólo uno de ellos se reconocía como indígena (del grupo mixteco). Los dos varones ingresaron a la primaria en el 2011 y recibían el estímulo económico de Arraigo y equidad E3. La maestra-directora ingresó a la escuela en 2014 y recibía un apoyo económico como directora de Tiempo Completo. Ella y uno de los maestros contaban con la Licenciatura en Educación para el Medio Indígena de la Universidad Pedagógica Nacional; el otro profesor había cursado la Licenciatura en Intervención Educativa en la misma universidad.

La primaria compartía el predio con una escuela preescolar, también del subsistema indígena. El plantel contaba con cuatro salones de clase. Tres de ellos habían sido construidos por los padres de familia. El otro егa un Aula Digna edificada por la Secretaría de Educación Pública. Había un espacio para la dirección escolar, una cancha de usos múltiples, un comedor con mesas al aire libre, otra cancha más pequeña y los baños. Esta escuela se encontraba en una ladera y la infraestructura se fue gestionando gradualmente parte de ella con recursos del PETc- por lo que podían observarse tres distintos niveles de construcción.

Los montos del financiamiento para esta escuela en el ciclo escolar 2018-2019 -con excepción del pago a los maestros y apoyos económicos por Tiempo Completo- fueron de 9,000 pesos del PIME. 40,000 pesos para gestión de infraestructura, 13 pesos diarios por estudiante para la comida y 3,800 pesos mensuales asignados a la madre de familia que fungía como coordinadora del comedor del PETC.

Además de estas cantidades, la directora mencionó que la escuela recibía algunos recursos en especie por parte de las autoridades educativas, como insumos para la impresora y dos copiadoras, así como productos para limpieza. El plantel también contaba con un espacio para la venta de alimentos y dulces que eran ofrecidos por una madre de familia durante el recreo escolar. Esta persona pagaba una cuota de 150 pesos 
semanales a la escuela. Con este dinero podía cubrirse el monto de otros gastos del funcionamiento de la cocina del comedor.

Según el testimonio del equipo docente, el $80 \%$ del alumnado es de ascendencia indígena. Alrededor de un $30 \%$ de los niños domina la lengua mixteca y otro 15\% tiene un dominio intermedio de la misma. La escuela realiza actividades para prestigiarla mediante su uso simbólico en eventos cívicos, como las ceremonias de honores a la bandera de cada lunes. En ellas se cantaba el himno nacional en español y mixteco. También, cada grupo tenía asignado un día de la semana para impartir la asignatura de lengua. En clases, los maestros buscaron el apoyo de algunos estudiantes (y padres de familia) que conocían la lengua para tratar temas relacionados con su enseñanza. Como en el caso de la escuela de Tijuana, no existía un modelo pedagógico oficial acorde a la escuela multigrado.

Durante el período de observación, la mayor parte de los días la escuela acató el horario reglamentario. Los días en los que esto no ocurrió tuvo que ver con la asistencia de los docentes a cursos de capacitación propuestos por el sistema educativo. En el mes de noviembre, los tres maestros de la primaria, junto a la profesora del preescolar, debieron acudir a Tijuana a tomar un curso de capacitación, motivo por el cual la escuela no impartió clases durante tres días hábiles. En otra ocasión, los maestros de primero y tercer ciclo debieron dejar el grupo temprano para trasladarse a la supervisión escolar a realizar un trámite administrativo.

En la segunda estancia en la escuela, la matrícula registrada se mantenía en 71 alumnos. El equipo docente también permaneció sin cambios. Fue notable que para el trabajo administrativo relacionado con el funcionamiento del programa y del PETc, favorecía el hecho de que la directora estuviera liberada de grupo después de mediodía al ser sustituida por el maestro contratado por el programa. Esto otorgaba mayores condiciones para que no se afectara el tiempo de enseñanza.

En suma, el tipo de gestión en esta escuela, por una parte, afectaba las condiciones para la enseñanza, atravesadas por una carga excesiva de cursos de capacitación y los requerimientos administrativos de la supervisión. Sin embargo, por otro lado, el que los tres maestros tuvieran una plaza base y que se contara con el personal de apoyo pagado por el PETC, contribuyó a mejores condiciones para la enseñanza que en la escuela de Tijuana.

\section{Discusión}

En las escuelas observadas se confirma lo ya ampliamente documentado para otras regiones del país e incluso del mundo: son planteles en alta vulnerabilidad; carecen de un modelo pedagógico oficial acorde a las aulas multigrado; deben responder a las mismas exigencias administrativas que las escuelas graduadas; carecen de suficientes maestros y a los que hay no se les proporcionan opciones de formación congruentes con las exigencias de la modalidad (Little, 1995; Mandujano, 2006; Schmelkes y Águila, 2019; Weiss et al., 2007). No obstante, las escuelas observadas -a diferencia de otras instituciones en regiones apartadas- se encuentran en zonas cercanas a centros urbanos. Los maestros viven relativamente cerca de los planteles, de manera que pueden trasladarse todos los días por la mañana y regresar a su casa por la tarde. Otra particularidad es que se encuentran en una zona fronteriza y turística (lo último sólo en el caso de la escuela en Ensenada), lo que impacta los procesos socioculturales entre los distintos actores de la comunidad escolar.

En términos de gestión, encontramos que la pertenencia al PETc ha beneficiado a ambas comunidades escolares, que han podido gestionar la construcción y equipamiento de cocinas-comedores adecuados y proporcionar un servicio de alimento al mediodía a los estudiantes, el cual es preparado por una madre de familia asignada como coordinadora del comedor. De forma gradual, durante los cuatro ciclos escolares que estuvieron inscritas en el programa, la infraestructura de las escuelas fue mejorada. No obstante, al mismo tiempo, la participación en el PETc trajo consigo una mayor carga administrativa, de capacitación y rendición de cuentas. Al no existir la figura de director con nombramiento en las escuelas multigrado, las funciones asignadas por el PETC recaen en la persona nombrada como directora, quien, en el caso de Tijuana, era responsable de un grupo. Entre sus funciones estaban la compra y el traslado de los alimentos 
a la escuela los fines de semana y la negociación de los pagos a los proveedores, ya que los recursos económicos del programa suelen retrasarse.

Por otro lado, la entrega del monto de los recursos para la gestión escolar no fue cumplida a cabalidad para la escuela de Ensenada. Mientras que la de Tijuana recibió los 90 mil pesos conforme las Reglas de Operación del PETC (Acuerdo 21/12/17), la de Ensenada sólo obtuvo la mitad del monto. En la primera, la mejora en la infraestructura fue notable, en la segunda no fue así. Como Weiler (como se citó en Ezpeleta, 2004, p. 421) señala, la formulación de programas puede procurar al estado beneficios políticos ante los gobernados. No obstante, la fase de la implementación es menos visible y puede irse abandonando gradualmente.

En ambos planteles la población tenía un vínculo fuerte con la escuela y los padres de familia jugaban un rol decisivo en su gestión. También se observó ausentismo entre los estudiantes. En la escuela de Tijuana esto estuvo relacionado con el parentesco entre los niños de uno y otro grupo. Cuando un grupo, por alguna razón, no tenía clases, los niños emparentados del otro grupo también faltaban. En la primaria de Ensenada el ausentismo estuvo relacionado con enfermedades de los niños vinculadas al descenso de las temperaturas. En las dos escuelas, los docentes subrayaron retrasos y materiales educativos incompletos, como libros de texto para estudiantes y para maestros.

Tras precisar características comunes en las dos escuelas, la tabla I enlista los rasgos distintivos de cada una.

Tabla I. Comparativo de aspectos escolares que inciden en la gestión pedagógica

\begin{tabular}{|c|c|c|}
\hline Aspecto de la escuela & Primaria multigrado en Tijuana & Primaria multigrado en Ensenada \\
\hline $\begin{array}{l}\text { Variación de la matrícula } \\
\text { (primera/segunda estancias) }\end{array}$ & $53 / 75$ & $71 / 71$ \\
\hline $\begin{array}{l}\text { Número de docentes/ condición } \\
\text { laboral }\end{array}$ & 2 Interinos & 3 Plaza Base \\
\hline $\begin{array}{l}\text { Número de docentes que se } \\
\text { reconocían a sí mismos como } \\
\text { indígenas }\end{array}$ & 1 de 2 & 1 de 3 \\
\hline $\begin{array}{l}\text { Número de maestros de Tiempo } \\
\text { Completo y autonomía curricular }\end{array}$ & 0 & 4 \\
\hline $\begin{array}{l}\text { Número de días observados en los } \\
\text { que se cumplió con los horarios } \\
\text { del PETC }\end{array}$ & 4 de 14 & 8 de 14 \\
\hline Enseñanza de la lengua & No se enseñó. & $\begin{array}{l}\text { Cada maestro tenía asignado un tiempo } \\
\text { semanal para la asignatura de lengua } \\
\text { (mixteco). La lengua se retomaba en las } \\
\text { asambleas de los honores a la bandera y } \\
\text { en algunos eventos especiales. }\end{array}$ \\
\hline Convivencia escolar & $\begin{array}{l}\text { Hubo problemas de convivencia } \\
\text { significativos entre los } \\
\text { estudiantes. También hubo } \\
\text { tensiones entre el maestro y } \\
\text { algunos de sus alumnos. }\end{array}$ & $\begin{array}{l}\text { Hubo problemas de convivencia entre } \\
\text { los estudiantes. Agresiones verbales. } \\
\text { Sin embargo, el equipo docente } \\
\text { promovía un clima de comunidad entre } \\
\text { estudiantes y maestros e incluso con } \\
\text { egresados de la escuela, quienes } \\
\text { libremente podían ingresar en ella y } \\
\text { hacer uso de las áreas deportivas o las } \\
\text { computadoras. }\end{array}$ \\
\hline Ceremonias cívicas & No hubo & $\begin{array}{l}\text { Se realizaron los lunes con los honores } \\
\text { a la bandera. }\end{array}$ \\
\hline Infraestructura/conectividad & $\begin{array}{l}\text { Hubo mejoras notables en el } \\
\text { comedor escolar y en el } \\
\text { alumbrado de la escuela. No había } \\
\text { Internet. }\end{array}$ & $\begin{array}{l}\text { La infraestructura no tuvo cambios } \\
\text { significativos entre las dos estancias de } \\
\text { observación. La escuela contaba con } \\
\text { Internet por las gestiones llevadas a } \\
\text { cabo por el fundador de la comunidad. }\end{array}$ \\
\hline
\end{tabular}

Fuente: elaboración propia 
Entre estas dos escuelas destaca que el número, la condición laboral y la permanencia de los docentes en los planteles representó una gran diferencia con relación a su gestión y la generación de condiciones para la enseñanza. Como señala Ezpeleta (1992b), los maestros no sólo son personas que enseñan, su desempeño está atravesado por otras dimensiones -como la laboral-; son profesionales, pero también trabajadores asalariados. Coincidimos con la autora, cuya argumentación señala que la dimensión laboral no puede considerarse como una variable independiente de la dimensión pedagógica (Ezpeleta, 1997).

En la primaria ubicada en Ensenada, el equipo docente tiene varios años trabajando en esa escuela, además de que todos tienen una plaza definitiva. Dos maestros reciben una compensación económica por trabajar en una zona considerada como marginada. Por otro lado, una maestra funge como directora de Tiempo Completo -liberada de grupo-y recibe una compensación económica.

Estas condiciones no pedagógicas atraviesan la operatividad de la escuela y permiten que los profesores permanezcan en ella, con la posibilidad de tejer vínculos con la comunidad. Esto se observa, por ejemplo, en el hecho de que tanto el maestro de PETC como la maestra de inglés pertenecen a la comunidad. A partir del 2011, cuando los dos primeros maestros de este equipo docente llegaron a la escuela, la primaria fue creciendo en infraestructura y se construyó la mitad de lo que ahora constituye el plantel. Tras varios años de haber sido una escuela señalada con bajos resultados educativos, en 2014 dejó de serlo. Esto, consideramos, explica la mejora en los resultados de la prueba PLANEA citados más arriba.

Además, este escenario permite que la actividad académica de la escuela presente un mayor dinamismo que la de Tijuana. Aun así, del total de días observados, solo el $57 \%$ del tiempo se cumplió con el horario reglamentario. Los alumnos tienen mayor tiempo de trabajo en el aula. La enseñanza de la lengua mixteca no es enseñada como lengua materna, sin embargo, sí ocupa un lugar simbólico significativo. En el marco del programa de clubes escolares, el equipo docente conformó tres: uno de español, uno de matemáticas y otro de artes. Al haber acceso a Internet, los niños usan las computadoras y tabletas existentes en la escuela. Participan en ceremonias cívicas donde se prestigia la lengua indígena. También, la institución está inscrita en el programa de prevención contra adicciones "FORMA" del Instituto de Psiquiatría del Baja California y reciben, semanalmente, la visita de una psicóloga del programa. Esta profesional atiende a niños de los tres ciclos. En esta escuela existen problemas de convivencia significativos entre estudiantes, aunque no relacionado con la condición indígena. El equipo docente intenta crear un clima de comunidad con actividades recreativas compartidas y actividades de convivencia con música y baile durante los recreos escolares.

En suma, en este plantel, la gestión -relacionada con la planta docente, su formación y condiciones laborales, el financiamiento, el trabajo administrativo, la relación con padres de familia y la comunidad, la interacción con las autoridades educativas, etc.- de varios ciclos escolares, ha posibilitado mejores condiciones para el trabajo de enseñanza-aprendizaje.

En la escuela en Tijuana, tanto el director como la directora del PETc eran relativamente recién llegados a la primaria. Los dos estaban frente a grupo y su condición laboral era de maestros interinos. Al ser interinos no podían acceder a la compensación para docentes que trabajan en poblaciones lejanas.

En este plantel, al que, ciertamente, el difícil acceso no le favorece, el tiempo de enseñanza fue significativamente menor que el de la escuela en Ensenada. Del tiempo observado, sólo el $28.57 \%$ fue efectivo. No hubo maestros frente a grupo del PETC, salvo la propia directora. Durante la observación, no se realizó trabajo del programa de clubes escolares (los docentes comentaron que sí se habían realizado con anterioridad). Había una biblioteca, pero no se usó, y un salón de cómputo que sólo tenía una computadora. No se realizaron ceremonias cívicas y no se enseñó la lengua indígena.

Mandujano (2006) presenta una caracterización de la gestión en escuelas multigrado en Chile, en la que detalla que la escuela multigrado bidocente presenta una percepción de "inestabilidad máxima", "con bajas posibilidades de innovación" y en donde "la escuela ni la comunidad pueden hacer mucho" (pp. 13-14). Al tener que dar cuenta de las responsabilidades de la dirección y del aula multigrado, los docentes de la escuela en Tijuana entraban en alto grado de agobio. A continuación, el testimonio del maestro-director: 
(Lo que se necesita es que) el sistema nos apoye con un maestro que esté ocupado en la dirección... porque no podemos cumplir con los aprendizajes esperados... o con los objetivos y metas que nosotros planteamos en nuestras planeaciones porque estoy a cargo de dirección [...] vienen mamás, vienen a preguntar o vienen visitas y como director los tengo que atender y en esa parte estoy descuidando a mis alumnos. Eso no nos permite cumplir con lo que se plantea... es muy difícil tener dos cargos... nos piden entrega de formatos, entrega de archivos... reuniones de directores... capacitaciones... entonces son cosas que tenemos que estar al tanto y no nos deja trabajar como se debe. (Maestro-director escuela de Tijuana, comunicación personal, 6 de junio de 2019).

A pesar de la sobrecarga de trabajo referida por el maestro, los dos docentes se esforzaron por gestionar recursos que consideraron podrían beneficiar a la comunidad escolar. Padres de familia refirieron con satisfacción la visita que policías municipales habían realizado con anterioridad a la escuela. Los agentes acudieron a impartir una plática a los estudiantes. Esta visita fue solicitada por el director como medida para que la convivencia con los niños mejorara, ya que, al igual que en la escuela de Ensenada, había problemas significativos de convivencia entre estudiantes.

Aunque ambos maestros establecieron vínculos profesionales y afectivos con la escuela y la comunidad, había posibilidades de que tuvieran que dejarla al final del ciclo escolar. Esto debido a su contratación de interinos. Existen tres docentes con plazas base asignados a la primaria, sin embargo, dos de ellos tienen licencia sin goce de sueldo y otro enfrenta un proceso jurídico, lo que condena a la escuela a una constante rotación de profesores con el impacto en las condiciones para la enseñanza que conlleva y que se vincula con el detrimento de los resultados de PLANEA. Esto no es privativo de esta escuela, se ha registrado que la rotación de maestros en las escuelas multigrado es un problema generalizado. A continuación, el testimonio de la madre de familia coordinadora del comedor:

Yo sé de esa situación de la escuela, de que está ocupado el lugar sin estar los profesores y eso nos viene a afectar a nosotros porque ellos (los interinos) se van... porque si hubiera ese lugar se quedarían porque ya saben cómo trabajar aquí en la escuela. (Comunicación personal, madre de familia, 7 de junio de 2019).

Como lo ejemplifica el testimonio de la madre de familia, el que un maestro deje la escuela significa volver a empezar para la comunidad, que ya ha visto transitar a muchos docentes en pocos años. Mandujano (2006) señala que, en el caso de las escuelas rurales multigrado, la salida de un profesor puede convertirse en una tragedia para la comunidad. Cada vez que un equipo docente se desintegra se interrumpe el trabajo pedagógico.

En las primeras observaciones en esta escuela notamos nerviosismo entre docentes y padres de familia debido a la disminución de la matrícula, lo cual podría originar el cierre de la misma. Algunas madres habían decidido sacar a sus niños de ese plantel y se afirmaba que era consecuencia del mal desempeño de la maestra que atendía los grados de primero a tercero. En una junta con el director y algunas madres de familia, una de ellas enfatizó que se había trabajado mucho para tener una escuela en la localidad, y que muchas madres consideraban buscar otra institución ante la falta de resultados educativos adecuados. En esta junta, el director respondió que se había decidido, junto con la supervisión escolar, no renovar el contrato a la profesora.

En la segunda estancia la matrícula había tenido un significativo incremento al pasar de 53 a 75 estudiantes registrados y los maestros lo atribuyeron al cambio de la docente. Este número les permitió solicitar un tercer maestro para la escuela ante el sistema educativo; sin embargo, esto no se concretó durante ese año lectivo.

En cambio, la matrícula de la escuela en Ensenada se mantuvo estable. Al tratarse de una población mayoritariamente de jornaleros agrícolas, algunos de ellos migrantes, los docentes deben en algunos casos adecuarse a los calendarios de trabajo de familias que migran a Oaxaca y luego regresan. Los estudiantes de estas familias no son dados de baja, se espera a que regresen y retomen su formación en la primaria. 


\section{Conclusiones}

El estudio de caso que presentamos permite constatar que para una mejora de los resultados de PLANEA, meta de la política educativa, es necesario considerar una mejora en aspectos no pedagógicos de la gestión.

Las dos escuelas observadas presentan variaciones significativas en cuanto al servicio que prestan en las comunidades escolares. Encontramos que esto se vincula, principalmente, con el número de maestros en las escuelas, así como con sus condiciones laborales y permanencia. También es importante subrayar el impacto en el tiempo de enseñanza que tienen los cursos de capacitación docente y las tareas administrativas impuestas por las autoridades estatales.

El estudio permitió confirmar la ausencia de un modelo pedagógico para las escuelas multigrado. Asimismo, subrayar cómo la gestión promovida no toma en cuenta las especificidades de la organización multigrado.

En el plano teórico-metodológico, la observación sobre los procesos de la gestión nos permitió destacar los componentes no pedagógicos que inciden en cada plantel, y constatar que, a pesar de que las escuelas comparten algunas semejanzas, esos componentes no pedagógicos inciden en los resultados educativos. También confirmamos que la gestión puede estar atravesada por las acciones de distintos actores y no sólo por los estudiantes, los maestros o las autoridades educativas.

Concluimos que esta perspectiva de investigación otorga significativas posibilidades para la comprensión de las distintas realidades educativas. Consideramos que, para el caso de las escuelas del sistema del servicio indígena en México, cuyos estudios se han enfocado mayormente en aspectos del derecho a la educación, la enseñanza de la lengua y la interculturalidad, el aporte del estudio de la gestión permite un conocimiento más detallado de diferentes factores que inciden en los procesos de aprendizaje.

El registro de la operación del PETC en estas dos escuelas evidencia cómo la inyección de recursos económicos y de servicio de alimentación a las escuelas es, sin duda, una política necesaria y en beneficio, sobre todo, de las comunidades más vulnerables. Sin embargo, sostenemos que si esta política no es constante en el cumplimiento de su propia normatividad y no considera las otras dimensiones que atraviesan la gestión, no se generarán las condiciones para cumplir con el objetivo de mejorar los procesos de la enseñanza y el aprendizaje.

Cabe subrayar el carácter exploratorio de esta investigación y la necesidad de indagar más sobre los aspectos que atraviesan la gestión en las escuelas, sobre todo lo relacionado con la permanencia y rotación de los docentes.

Por último, nos sumamos a las recomendaciones de elaborar políticas educativas que tomen en cuenta las particularidades de la escuela multigrado, tanto en sus aspectos pedagógicos como de gestión. Estas políticas deberían buscar alternativas de solución a los añejos problemas de la falta de maestros en condiciones laborales adecuadas que les permitan permanecer en las escuelas. Con esto, argumentamos, se estarían generando mejores condiciones para la enseñanza de las poblaciones más vulnerables.

\section{Referencias}

Acuerdo número 21/12/17 por el que se emiten las Reglas de Operación del Programa de Escuelas de Tiempo Completo para el ejercicio fiscal 2018. Diario Oficial de la Federación, México, 26 de diciembre de 2017.

Alfirevic, N., Burusic, J., Pavicic, J. y Relja, R. (Eds.) (2016). School effectiveness and educational management: towards a south-eastern europe research and public policy agenda. Palgrave macmillan. 
Arriagada, C. R. y Calzadilla, O. (2017). Percepción de las bases curriculares de la educación básica multigrado en la Araucanía, Chile. Praxis \& Saber, 9(20), 75-95.

https://doi.org/10.19053/22160159.v9.n20.2018.8296

Blease, B. y Condy, J. (2015). Teaching of writing in two rural multigrade classes in the Western Cape. Reading \& Writing, 6(1), 1-9. https://doi.org/10.4102/rw.v6i1.58

Bonilla, O. (2008). Gestión escolar en México. Algunos aprendizajes. En J. Gairín y S. Antúnez (Coords.), Organizaciones educativas al servicio de la sociedad (pp. 1-17). Wolters Kluwer.

Butsankom, A., Sirishuthi, C. y Lammana, P. (2016). The development effectiveness management model for sub-district secondary school. Educational Research and Reviews, 11(19), 1790-1804.

https://doi.org/10.5897/ERR2016.2849

Caldwell, B. J. (2005). School-based management, education policy series. UNESCO.

Ezpeleta, J. (1992a). Problemas y teoría a propósito de la gestión pedagógica. En J. Ezpeleta y A. Furlán (Comps.), La gestión pedagógica de la escuela. UNESCO/OREALC.

Ezpeleta, J. (1992b). El trabajo docente y sus condiciones invisibles. Nueva Antropología, 2(47), 27-47. https://revistas-colaboracion.juridicas.unam.mx/index.php/nuevaantropologia/article/view/14645/13059

Ezpeleta, J. (1997). Algunos desafíos para la gestión de las escuelas multigrado. Revista Iberoamericana de Educación, 15, 101-120. https://rieoei.org/historico/oeivirt/rie15a04.htm

Ezpeleta, J. (2004). Innovaciones educativas. Reflexiones sobre los conceptos en su implementación. Revista Mexicana de Investigación Educativa, 9(21), 403-424.

https://www.comie.org.mx/revista/v2018/rmie/index.php/nrmie/article/view/853/853

Galván, L. y Espinoza, L. (2017). Diversidad y prioridades educativas en escuelas multigrado. Estudio de caso en México. Sinéctica, 49, 1-19. https://sinectica.iteso.mx/index.php/SINECTICA/article/view/715

Gertler, P., Patrinos, H. A., Rubio-Codina, M. (2007). Impact evaluation for school-based management reform. Informe no. 10. The World Bank. http://documents.worldbank.org/curated/en/

671361468313736152/Impact-evaluation-for-school-based-management-reform

Githiari, F. (2017). Ways through which principals acquire the leadership competencies required for effective management of secondary schools in Nairobi County, Kenya. Journal of Education and Practice, 8(9), 43-48. https://www.iiste.org/Journals/index.php/JEP/article/view/36057/37052

Instituto Nacional para la Evaluación de la Educación. (2019). Panorama educativo de México 2018. Indicadores del Sistema Educativo Nacional. Educación básica y media superior. https://www.inee.edu.mx/wpcontent/uploads/2019/08/P1B117.pdf

Instituto Nacional para la Evaluación de la Educación. (s.f.). Evaluación de Condiciones Básicas para la Enseñanza y el Aprendizaje (ECEA). Base de datos. Primaria 2014.

https://www.inee.edu.mx/evaluaciones/ecea/bases-de-datos-primaria-2014/

Little, A. (1995). Multigrade teaching: a review of practice and research (serial No. 12). Overseas Development Administration.

Little, A. (2004). Learning and teaching in multigrade settings. Paper commissioned for the EFA Global Monitoring Report 2005, The Quality Imperative. http://multigrade.ioe.ac.uk/fulltext/fulltextLittle.pdf 
Mandujano, F. (2006). La gestión de las escuelas rurales multigrado I. Revista digital eRural, Educación, cultura y desarrollo rural, 7, 1-17.

Mejía, F., Argándar, E., Arruti, M., Olvera, A. y Estrada, M. (2016). Programa de aprendizaje en multigrado: una experiencia de mejora educativa en el estado de Puebla. Revista Latinoamericana de Estudios Educativos, 46(3), 111-136. https://www.cee.edu.mx/rlee/revista/r2011-2020/r texto/t 20163 05.pdf

Mortimore, P., Sammons, P., Stoll, L., Lewis, D. y Rusell, J. E. (1988). School matters: the junior years. The University of California Press.

Naidoo, P. (2019). Perceptions of teachers and school management teams of the leadership roles of public school principals. South African Journal of Education, 39(2), 1-14.

https://doi.org/10.15700/saje.v39n2a1534

Nava, M. V. (Coord.) (2018). Gestión escolar, liderazgo y gobernanza. Construcciones, deconstrucciones y retos en instituciones de educación obligatoria. Secretaría de Educación Pública.

Parcerisa, L. (2016). Nueva gestión pública y reforma educativa: la recontextualización de la autonomía escolar en diferentes contextos escolares en Cataluña. Revista Portuguesa de Educação, 29(2), 359-390. https://doi.org/10.21814/rpe.7928

Rockwell, E. (2009). La experiencia etnográfica. Historia y cultura en los procesos educativos. Paidós.

Schmelkes, S. y Águila, G. (Coords.). (2019). La educación multigrado en México. Instituto Nacional para la Evaluación de la Educación.

Secretaría de Educación Pública. (2020). PLANEA en educación básica. http://planea.sep.gob.mx/ba/

Secretaría de Educación Pública. (s.f.). PLANEA. Localización geográfica de escuelas primarias. Resultados 2018. https://primarias.resultadosplanea.connectit.company/map?state=2

SEP-Subsecretaría de Educación Básica-DGEI. (2019). Prontuario de la estadística de la educación indígena nacional, 2017-2018. https://dgei.basica.sep.gob.mx/es/prontuarios-estadisticos/2017-2018/pe 20172018.html

Smyth, J. (Ed.) (2001). La autonomía escolar: una perspectiva crítica. Ediciones Akal

Tolofari, S. (2005). New public management and education. Policy Futures in Education, 3(1), 75-89. https://doi.org/10.2304/pfie.2005.3.1.11

UNESCO. (2015). Practical tips for teaching multigrade classes. Embracing diversity: toolkit for creating inclusive, learning-friendly environments. Specialized Booklet 4.

https://unesdoc.unesco.org/ark:/48223/pf0000220101

UNESCO e International Institute for Education Planning. (2019). School leadership and management. Learning portal. https://learningportal.iiep.unesco.org/en/issue-briefs/improve-learning/schools-and-classrooms/ school-leadership-and-management

Weiss, E., Block, D., Candela, A., Pellicer, A., Taboada, E. y Rockwell, E. (2007). Evaluación externa de la construcción de la propuesta educativa multigrado 2005 (Reporte final). Centro de Investigación y Estudios Avanzados del Instituto Politécnico Nacional. http://departamentos.cinvestav.mx/Portals/die/SiteDocs/ Investigadores/EWeiss/EMultigrado/EvaluacionExternadelaConstrucciondelaPEM2005.pdf 